\title{
SIP30 Is Required for Neuropathic Pain-Evoked Aversion in Rats
}

\author{
Mei Han (韩梅), ${ }^{1}$ Xiao Xiao (肖晓), ${ }^{1}$ Yan Yang (杨艳), ${ }^{1}$ Ru-Yi Huang (黄如一), ${ }^{2}$ Hong Cao (曹红), ${ }^{1}$ \\ Zhi-Qi Zhao (赵志奇), ${ }^{1}$ and Yu-Qiu Zhang (张玉秋) ${ }^{1}$ \\ ${ }^{1}$ Institute of Neurobiology, Institutes of Brain Science and State Key Laboratory of Medical Neurobiology, Fudan University, Shanghai 200032, China, and \\ ${ }^{2}$ Department of Life Science, Fudan University, Shanghai 200433, China
}

SIP30 (SNAP25 interacting protein of 30) is a SNAP25 interaction protein of $30 \mathrm{kDa}$ that functions in neurotransmitter release. Using a chronic constriction injury (CCI) model of neuropathic pain, we profiled gene expression in the rat spinal cord and brain and identified sip30, which was upregulated after CCI. Here, we show that CCI induced a bilateral increase of SIP30 in the rostral anterior cingulate cortex (rACC), a key brain region that has been implicated in pain affect. We put rats in a chamber with one half painted white (light area) and the other half painted black (dark area), and measured neuropathic pain-evoked place escape/avoidance paradigm (PEAP) to quantify the level of negative emotion evoked by painful stimuli using a Von Frey hair. Inhibition of CCI-mediated induction of SIP30 by intra-rACC injection of shRNA targeting the rat sip30 gene reduced PEAP. Interestingly, knockdown of SIP30 did not affect CCI-induced evoked pain such as heat hyperalgesia and mechanical allodynia. Neither did it affect general learning and memory. CCI-induced upregulation of SIP30 was correlated with activation of ERK, PKA, and CREB in the rACC. Intra-rACC administration of PKA or ERK inhibitors suppressed CCI-induced SIP30 upregulation and blocked the induction of PEAP. Additionally, knockdown of SIP30 suppressed the frequency of mEPSCs and increased paired-pulse ratios in rACC slices and decreased extracellular glutamate concentrations. Together, our results highlight SIP30 as a target of PKA and ERK in the rACC to mediate neuropathic pain-evoked negative emotion via modulation of glutamate release and excitatory synaptic transmission.

Key words: anterior cingulate cortex; chronic constriction injury; neuropathic pain; pain-related negative emotion; place escape/avoidance paradigm; SIP30

\section{Introduction}

Peripheral nerve injury not only induces hypersensitivity to cutaneous stimuli such as hyperalgesia and allodynia but also causes affective disturbances (Mutso et al., 2012; Yu et al., 2013). Remarkably, emotional components of pain are far less studied than the sensory components (Auvray et al., 2010). Although these emotional components are widely acknowledged to worsen the quality of life for a large subset of neuropathic pain patients, the biological bases of affective changes remain poorly understood.

Accumulating evidence implicates that the anterior cingulate cortex (ACC) in the affective response to noxious stimuli (Price, 2000). Previous studies have demonstrated that the destruction of neurons originating from the rostral ACC (rACC) blocks formalin-induced conditioned place avoidance (CPA), a pain-

\footnotetext{
Received July 26, 2013; revised Nov. 4, 2013; accepted Nov. 7, 2013.

Author contributions: M.H. and Y.-Q.Z. designed research; M.H., X.X., Y.Y., R.-Y.H., and H.C. performed research; M.H., X.X., Z.-Q.Z., and Y.-Q.Z. analyzed data; M.H. and Y.-Q.Z. wrote the paper.

This work was supported by the National Basic Research Program of China (Grant 2013CB531905) and the National Natural Science Foundation of China (Grants 31121061, 31070973, 31271183, and 31371123). We thank Dr. R.J. Wenthold for providing SIP30 antibodies. We also thank Dr. Rurong Ji and Dr. David Saffen for their critical reading and linguistic edit of the manuscript.

The authors declare no competing financial interests.

Correspondence should be addressed to Dr. Yu-Qiu Zhang, Institute of Neurobiology, Fudan University, 131 Dong An Road, Shanghai 200032, China. E-mail: yuqiuzhang@fudan.edu.cn.

DOI:10.1523/JNEUROSCI.3160-13.2014

Copyright $\odot 2014$ the authors $\quad 0270-6474 / 14 / 340346-10 \$ 15.00 / 0$
}

related avoidance learning model, in rats without reducing acute pain-related behaviors (Johansen et al., 2001; Gao et al., 2004). Direct activation of rACC neurons can produce CPA in the absence of a peripheral noxious stimulus, suggesting that neurons in the rACC mediate both pain-related negative affect and nociceptor-driven aversive teaching signal (Johansen and Fields, 2004). Moreover, previous studies from our laboratory revealed that activation of NMDA receptors in the rACC is necessary for formalin pain-induced negative emotion (Lei et al., 2004; Ren et al., 2006; Li et al., 2009). Furthermore, activation of extracellular signal-regulated kinase 1 and 2 (ERK1/2) and subsequent phosphorylation of cAMP response element-binding protein (CREB) is implicated in this process (Cao et al., 2009, 2012). However, the identities of CREB target genes in the rACC and the role of these genes in affective pain remain to be investigated.

SIP30 was first identified in the cochlear hair cells of the organ of Corti of guinea pigs (Lee et al., 2002). This novel protein, like all soluble $N$-ethylmaleimide-sensitive factor (NSF) attachment protein receptors (SNAREs), has a coiled-coil domain that forms a key component of protein-protein interactions (Mochida, 2000). SNAREs, including target SNAREs and vesicular SNAREs, are essential for the regulated exocytosis of synaptic vesicles during neurotransmission (Bennett et al., 1992; Söllner et al., 1993). SIP30 has been detected in various brain areas (Lee et al., 2002; Marie-Claire et al., 2007). Through differential screening of rat 
brain cDNA libraries, we identified sip30 as a gene that is upregulated in the CNS in response to neuropathic pain (Zhang et al., 2009). We also found that upregulation of SIP30 in the spinal cord in rats with chronic constriction injury (CCI) of the sciatic nerve can be suppressed by intrathecal delivery of an MEK inhibitor that blocks the phosphorylation of the ERKs. In PC12 cells, upregulation of SIP30 by nerve growth factor (NGF) is also dependent on ERK activation. We have shown that there is an ERKresponsive region in the rat sip30 promoter, and activation of ERK promotes the recruitment of the transcription factor CREB to the sip30 gene promoter (Peng et al., 2009).

In this study, we hypothesized that SIP30 in the rACC, as a downstream target of the ERK-CREB signaling pathway, might contribute to neuropathic pain-evoked negative emotion.

\section{Materials and Methods}

Experimental animals. Male Sprague Dawley rats (220-250 g; Experimental Animal Center of Fudan University) were maintained on a $12 \mathrm{~h}$ light/dark cycle with food and water available ad libitum. CCI of the sciatic nerve was performed according to previous protocols (Zhang et al., 2009). After receiving anesthesia with pentobarbital (45 mg/kg, i.p.), the right sciatic nerve was exposed at the mid-thigh level, and four chromic gut (4-0) ligatures were tied loosely around the nerve $\sim 1 \mathrm{~mm}$ apart, proximal to its trifurcation. For sham group rats, the sciatic nerve was isolated without ligation. All experimental procedures were approved by the Shanghai Animal Care and Use Committee and followed the ethical guidelines of the International Association for the Study of Pain regarding the use of laboratory animal (Zimmermann, 1983). All behavioral tests, quantification of Western blots, and immunohistochemical experiments described herein were performed by experimenters that were blind to the treatments.

shRNA lentivirus vector of the sip30 gene and intra-rACC infusions. shRNA targeting the rat sip30 gene (shSIP30; GenBank accession no. BC063144) and nontargeting control shRNA (shNC) sequences were designed and synthesized by Shanghai GeneChem Co., Ltd. The sequence targeting sip30 and the nontargeting negative control were 5'-GAGGCACACAGGAAGTACA-3' and 5'-TTCTCCGAACGTGTCACGT-3', respectively. The recombinant virus coexpressing GFP was packaged using a Lentivector Expression Systems kit (Shanghai GeneChem Co., Ltd).

Rats were anesthetized and placed into a stereotaxic device with bregma and lambda at the same horizontal level. A 30-gauge stainless steel guide cannula with a 33-gauge stainless steel stylet plug was bilaterally inserted $0.5 \mathrm{~mm}$ above the rACC injection site [from bregma: anteroposterior, $2.7 \mathrm{~mm}$; mediolateral, $\pm 0.6 \mathrm{~mm}$; dorsoventral, $-2.5 \mathrm{~mm}$ ] according to the atlas of Paxinos and Watson (1998). Microinjections were performed through a 33-gauge stainless steel injection cannula that extended $0.5 \mathrm{~mm}$ beyond the tip of the guide cannula. A volume of $1 \mu \mathrm{l}$ $\left[1.5 \times 10^{9}\right.$ transducing units (TU) $]$ per hemisphere of either shRNA or shNC was injected over a 5 min period. The injection cannula was left in place for an additional 5 min to minimize the spread of the liquid along the injection track. The transfection efficiency of the lentivirus was observed at 7 and $14 \mathrm{~d}$ after infection.

Place escape/avoidance paradigm. Place escape/avoidance paradigm (PEAP) testing was conducted as described previously (LaGraize and Fuchs, 2007). Individual rats were placed in a $50 \times 30 \times 30 \mathrm{~cm}$ chamber on top of a raised mesh floor with one half painted white (light area) and the other half painted black (dark area). Animals were allowed unrestricted movement throughout the test chamber. A suprathreshold mechanical stimulus ( $60 \mathrm{~g}$, Von Frey filament) was applied to the plantar surface of the hindpaws at $15 \mathrm{~s}$ intervals throughout the $30 \mathrm{~min}$ test period. The mechanical stimulus was applied to the affected paw (ipsilateral to the CCI) while the animal was located in the dark area, and the naive paw (contralateral to the CCI) was stimulated when the animal was in the light area. This test assesses whether the noxious stimulation is sufficiently bothersome for the animal to escape/avoid the preferred dark area and uses the amount of time spent on the nonpreferred light side to quantify the level of pain affect (Fuchs and McNabb, 2012).
Hargreaves' test. Thermal hyperalgesia was assessed by measuring paw withdrawal latencies (PWLs) in response to a radiant heat source using Hargreaves' test. Briefly, with rats placed individually into Plexiglas chambers on an elevated glass platform, under which a radiant heat source (IITC Life Science) was applied to the glabrous surface of the paw through the glass plate. The heat source was turned off when the rat lifted the foot, allowing the measurement of the PWL. A 20 s cutoff was used to prevent tissue damage in the absence of a response.

Von Frey test. Mechanical allodynia was assessed by measuring paw withdrawal thresholds (PWTs) in response to a calibrated series of Von Frey hairs (Stoelting). Rats were placed individually into wire meshbottom cages. A series of calibrated Von Frey hairs were applied to the plantar surface of the hindpaw in ascending order $(2,4,6,8,10,15$, and $26 \mathrm{~g}$ ) with a sufficient force to bend the hair for $2 \mathrm{~s}$ or until paw withdrawal. A withdrawal response was considered valid only if the hindpaw was completely removed from the customized platform. Each hair was applied five times, and the minimal value that caused at least three responses was recorded as the PWT.

Conditioned place avoidance. The place conditioning apparatus and the CPA procedure have been described previously (Xiao et al., 2013). The place conditioning apparatus consisted of three opaque acrylic compartments (one neutral chamber and two conditioning chambers with distinct olfactory, visual, and tactile cues). The experimental process consists of the following three distinct sessions: a preconditioning session; a conditioning session; and a test (postconditioning) session. $\mathrm{LiCl}-$ induced CPA ( $\mathrm{LiCl}-\mathrm{CPA}$ ) task processing required $4 \mathrm{~d}$. Day 1 was the preconditioning day. Individual rats were allowed to explore the two conditioning compartments freely for $15 \mathrm{~min}$. A timer automatically recorded the time spent in each of the compartments in a blind manner. Rats that spent $>80 \%$ (720 s) on one side on that day were eliminated from the subsequent experiments. Day 2 was the conditioning day. Rats received no treatment in the morning and were randomly confined to one of the conditioning compartments for $30 \mathrm{~min}$. After at least $4 \mathrm{~h}$, in the afternoon the rats received $\mathrm{LiCl}(150 \mathrm{mg} / \mathrm{kg}$, i.p.; Frisch et al., 1995; Rinaman et al., 2009) or normal saline (NS) and were restricted to the opposite conditioning compartment for $30 \mathrm{~min}$. On day 3 , trials identical to those of day 2 were repeated. Day 4 was the postconditioning day. The procedure was the same as that of day 1 . The time that the animals spent in each compartment was measured. Regarding the fear conditioninginduced CPA, the CPA procedure consisted of $3 \mathrm{~d}$. The pretest (day 1) and post-test (day 3 ) were identical to those of the LiCl-CPA. Day 2 was the conditioning day. On day 2 , in the morning, the rats received no treatment and were randomly confined to one of the conditioning compartments for $30 \mathrm{~min}$. After at least $4 \mathrm{~h}$, in the afternoon, the rats received electric footshocks ( $0.5 \mathrm{~mA}$ for $0.2 \mathrm{~s}$ ) every $8-10 \mathrm{~min}$ in a compartment that was opposite to that of the conditioning compartment during the 30 min training session. Compartments were counterbalanced across the different conditioning groups.

Immunohistochemistry. After defined survival times, the rats were killed with overdoses of urethane $(1.5 \mathrm{~g} / \mathrm{kg})$ and perfused transcardially with NS followed by $4 \%$ paraformaldehyde. Brains were postfixed in the same fixative for $2-4 \mathrm{~h}$ at $4^{\circ} \mathrm{C}$ and immersed in a $10-30 \%$ gradient of sucrose for $24-48 \mathrm{~h}$ at $4^{\circ} \mathrm{C}$ for cryoprotection. Coronal brain sections ( 30 $\mu \mathrm{m})$ were cut with a cryostat (1900, Leica) and processed for immunofluorescence. Briefly, the sections were blocked with $10 \%$ donkey serum in $0.01 \mathrm{M} \mathrm{PBS}$, pH 7.4, with $0.3 \%$ Triton $\mathrm{X}-100$ for $1 \mathrm{~h}$ at room temperature (RT), and incubated overnight at $4^{\circ} \mathrm{C}$ with rabbit anti-SIP30 (1: 1000; a gift from the laboratory of Dr. R.J. Wenthold, National Institutes of Health) primary antibody in PBS with $1 \%$ normal donkey serum and $0.3 \%$ Triton X-100. For SIP30/NeuN, SIP30/GFAP, SIP30/OX-42, SIP30/phosphorylated ERK (pERK), SIP30/pCREB, pPKA/pERK, pPKA/ pCREB, or pERK/pCREB double immunofluorescence, the sections were incubated with a mixture of rabbit anti-SIP30 (1:1000) and mouse antiNeuN (neuronal marker, 1:1000; Millipore), mouse anti-GFAP (astrocytic marker, 1:1000, Sigma), anti-OX-42 (microglial marker, 1:500; Serotec), anti-pERK (1:2000; Sigma), or anti-pCREB (1:2000; Millipore), or rabbit anti-pPKA substrate (1:100; Cell Signaling Technology) and mouse-anti pERK or anti-pCREB, or rabbit-anti pERK (1:200; Cell Signaling Technology) and mouse-anti-pCREB overnight at $4^{\circ} \mathrm{C}$. After 
three 15 min rinses in PBS, the sections were incubated in fluorescein isothiocyanate-conjugated donkey anti-rabbit (or anti-mouse) IgG (1:200; Jackson ImmunoResearch) or rhodamine-conjugated donkey anti-mouse (or anti-rabbit) IgG (1:200; Jackson ImmunoResearch) for $2 \mathrm{~h}$ at $4^{\circ} \mathrm{C}$, and then washed in PBS. All sections were coverslipped with a mixture of $50 \%$ glycerin in PBS and then observed with a Leica SP2 confocal laserscanning microscope. The specificities of the immunostaining were verified by omitting the primary antibodies, which resulted in the disappearance of the immunostaining signals. The specificities of primary antibodies were verified by preabsorption experiments. Sections were first incubated overnight with a mixture of the primary antibodies and the corresponding blocking peptide (5:1 blocking peptide/primary antibody), followed by incubation with a secondary antibody. The immunostaining signals were abolished after absorption.

Western blot. Rats were killed with overdoses of urethane. The rACC was quickly dissected on ice using a Rat Brain Matrix (Stoelting Company), and then the rACC tissues were then homogenized in lysis buffer (12.5 $\mu \mathrm{l} / \mathrm{mg}$ tissue) containing a mixture of protease inhibitors (Roche) and PMSF (Sigma). After incubating on ice for $30 \mathrm{~min}$, the samples were centrifuged at $10,000 \mathrm{rpm}$ for $15 \mathrm{~min}$ at $4^{\circ} \mathrm{C}$. The supernatants were used for Western blotting.

Equal amounts of protein $(\sim 20 \mu \mathrm{g})$ were loaded and separated in $10 \%$ Tris-Tricine SDS-PAGE gels. The resolved proteins were transferred onto PVDF membranes (GE Healthcare Bio-Science). The membranes were blocked in $10 \%$ nonfat milk for $2 \mathrm{~h}$ at RT and incubated overnight at $4^{\circ} \mathrm{C}$ with rabbit anti-SIP30 (1:5000), mouse anti-pERK, mouse antiERK or rabbit anti-pPKA RII (1:1000; Millipore) primary antibody. The blots were then incubated with the secondary antibody, goat anti-mouse or anti-rabbit $\operatorname{IgG}$ conjugated with horseradish peroxidase (1:1000; Pierce), for $2 \mathrm{~h}$ at $4^{\circ} \mathrm{C}$. Signals were finally visualized using enhanced chemiluminescence (Pierce), and the blots were exposed onto x-ray film. All Western blot analyses were performed at least three times, and consistent results were obtained.

For the quantification of Western signals, the density of specific bands was measured using the Bio-Rad Image Analysis System. The same size square was drawn around each band to measure the density, and the background near that band was subtracted. GAPDH was used as the internal control. SIP30 or pPKA level was normalized against GAPDH level and expressed as a fold increase compared with control. Since total ERK and CREB levels did not change significantly after CCI, we used total ERK or total CREB levels as loading control, and pERK1/pERK2 and pCREB levels were normalized against total ERK1/ERK2 and total CREB levels, respectively, and were expressed as the fold increase compared with control.

Microdialysis and HPLC. The microdialysis probe (MD2200, BASi) was inserted into the unilateral rACC via the guide cannula to $1 \mathrm{~mm}$ beyond the tip of the guide cannula. The dialysis probe was perfused with artificial CSF (ACSF) at a flow rate of $2 \mu \mathrm{l} / \mathrm{min}$ via a microinfusing pump (1.0 ml Microdialysis Syringe, BASi). After dialysate levels stabilized $(\sim 1$ h), four sequential 20 min samples were collected as baseline values, and then SIP30-shRNA or shNC was microinjected into the bilateral rACC. The guide cannula was plugged up with a sterile stainless steel stylet plug. Seven days later, dialysate samples were collected, and then CCI (or sham) surgery was performed. On days 3 and 7 after CCI (days 10 and 14 after SIP30-shRNA or shNC), dialysate samples were collected again. Aliquots were frozen at $-80^{\circ} \mathrm{C}$ for later analyses.

Glutamate concentrations were analyzed with reversed-phase HPLC (Finnigan, Thermo Electron) with fluorometric detection following precolumn derivatization with $o$-phthalaldehyde. Chromatography was performed on a reversed-phase $\mathrm{C}-18$ column using a $\mathrm{pH}$ sodium acetate methanol gradient. Methionine sulfone was added to each sample as an internal standard. External standards containing 40, 400, or 4000 $\mathrm{pmol} / 20 \mathrm{ml}$ glutamate was run at the beginning and the end of each sample group. Glutamate peak heights were initially normalized to the methionine sulfone peak and then quantified based on a linear relationship between peak height and the amounts of the corresponding standards.

Slice preparation and whole-cell recordings. Coronal brain slices containing the rACC were obtained from young rats ( $4-5$ weeks old). SIP30-
shRNA or shNC was microinjected into the rACC $7 \mathrm{~d}$ before the electrophysiological experiments. After anesthesia with isoflurane, rats were decapitated. The brains were quickly removed and submerged in preoxygenated $\left(95 \% \mathrm{O}_{2}, 5 \% \mathrm{CO}_{2}\right)$ cold ACSF at $4^{\circ} \mathrm{C}$ that contained 126 $\mathrm{mm} \mathrm{NaCl}, 4.0 \mathrm{~mm} \mathrm{KCl}, 1.25 \mathrm{~mm} \mathrm{MgCl}$, $26 \mathrm{~mm} \mathrm{NaHCO} 3,1.25 \mathrm{~mm}$ $\mathrm{NaH}_{2} \mathrm{PO}_{4}, 2.5 \mathrm{~mm} \mathrm{CaCl} 2$, and $10 \mathrm{~mm}$ glucose. The osmolarity was adjusted to $290-320 \mathrm{mOsmol} / \mathrm{L}$, and the $\mathrm{pH}$ was adjusted to 7.35 . A tissue block containing the rACC was glued to the stage using cyanoacrylate glue. Slices $(400 \mu \mathrm{m})$ were cut with a vibratome (VT 1000S, Leica) and transferred to an oxygenated chamber at RT $\left(24 \pm 1^{\circ} \mathrm{C}\right)$ for at least $1 \mathrm{~h}$ before further processing.

A single slice was then transferred to a recording chamber and continuously perfused with recording solution at a rate of $5 \mathrm{ml} / \mathrm{min}$ at RT. Whole-cell recordings were obtained from pyramid-shaped neurons in layer II-III with an Axon Axopatch 200B amplifier (Molecular Devices) under visual control using differential interference contrast and infrared optics via a water-immersion objective (DMLFSA, Leica) and a CCD camera (IR-1000E). Recording electrodes made from $1.5 \mathrm{~mm}$ glass capillaries were pulled on a Flaming-Brown micropipette puller (P-97, Shutter Instrument) and filled with an intracellular solution containing the following (in mM): 150 gluconate, $8 \mathrm{NaCl}, 0.4$ EGTA, 2 Mg-ATP, 0.3 Na3GTP, and 10 HEPES, with the $\mathrm{pH}$ adjusted to 7.2 and osmolarity adjusted to $300 \mathrm{mOsmol} / \mathrm{L}$. With this solution, the recording electrodes exhibited a resistance of 2-6 M $\Omega$. Signals were low-pass filtered at $2 \mathrm{kHz}$ and digitized at $5 \mathrm{kHz}$ with a digitizer (Digidata 1322 and 1440, Molecular Devices). Miniature EPSCs (mEPSCs) were recorded at a holding potential of $-70 \mathrm{mV}$ in the presence of tetrodotoxin $(0.5 \mu \mathrm{M})$ and picrotoxin $(50 \mu \mathrm{M})$ to block voltage-dependent sodium channels and $\mathrm{GABA}_{\mathrm{A}}$ receptors, respectively. In the paired-pulse ratio (PPR) experiments, the paired-pulse stimulation (interpulse intervals: $35-250 \mathrm{~ms}$ ) was delivered via a bipolar tungsten stimulating electrode placed in layer $\mathrm{V}$ of the rACC. The second EPSC (EPSC2), which was evoked by the second of the paired-pulse stimulations, was compared with the EPSC1. Series resistance (Rs) was monitored during recording. Cells in which the Rs varied by $>20 \%$ and the Rs $>60 \mathrm{M} \Omega$ were excluded from analyses. Data were collected with pClamp9.2 software and analyzed using Clampfit 9.2.

Statistical analysis. The data are presented as the mean \pm SEM. Student's $t$ tests, paired $t$ tests, and one-way or two-way ANOVA followed by post hoc Student-Newman-Keuls tests were used to identify significant differences. In all cases, $p<0.05$ was considered to be statistically significant.

\section{Results}

\section{Peripheral nerve injury upregulated SIP30 in the rACC}

Baseline measures of PWLs in response to radiant heat stimulation and PWTs in response to Von Frey hairs from both hindpaws did not differ between the CCI and sham CCI groups before surgery. Strong thermal hyperalgesia and mechanical allodynia stably occurred in the ipsilateral hindpaw on day 3 and persisted over $14 \mathrm{~d}$ after CCI (two-way ANOVA; Groups: PWL, $F_{(3,24)}=$ 186.885, $p<0.01$; PWT, $F_{(3,24)}=64.424, p<0.001$; Group $\times$ Time interaction: PWL, $F_{(12,96)}=10.973, p<0.01$; PWT, $F_{(2,96)}=$ 4.921, $p<0.01)$. There were no significant differences in PWLs and PWTs between he hindpaws of the sham rats and the contralateral hindpaw of CCI rats before or after surgery (Fig. 1A,B). CCI induced a robust upregulation of SIP30 protein in the bilateral rACC (one-way ANOVA; ipsilateral: $F_{(6,35)}=14.793, p<$ 0.01 ; contralateral: $F_{(6,35)}=12.479, p<0.01$; Fig. $\left.1 C, D\right)$. Immunohistochemistry revealed SIP30-positive cells in laminae II-VI of the rACC (Fig. 1E). Double immunofluorescence showed that nearly all of the SIP30-positive cells in the rACC were neurons. SIP30 immunoreactivity was prevalent in the cytoplasm of the cell body and dendrites. SIP30 staining did not overlap staining for either the astrocytic marker GFAP or the microglial marker OX-42 (Fig. 1F). 
A

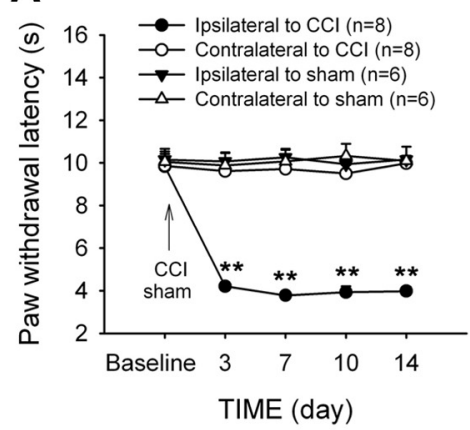

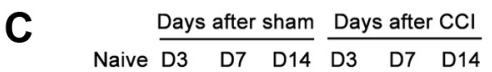
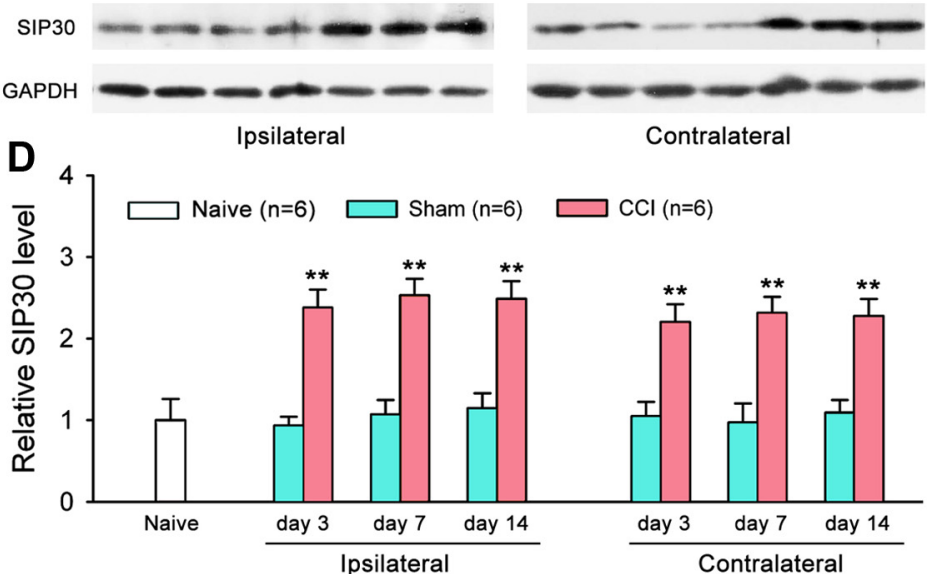

$\mathbf{E}$

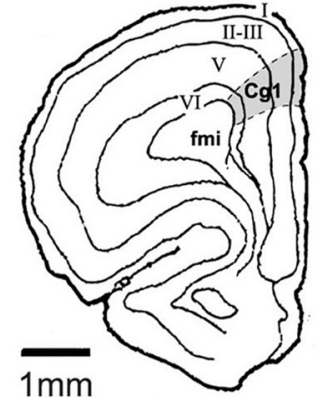

B

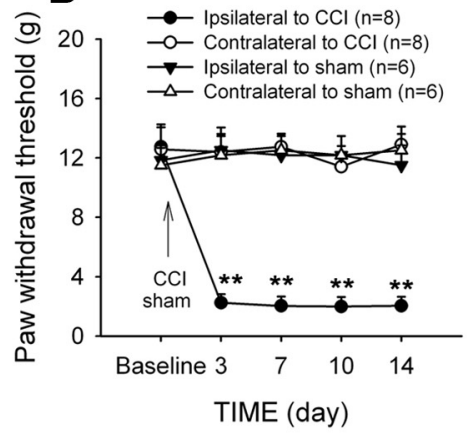

Days after sham Days after $\mathrm{CCl}$

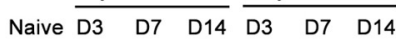

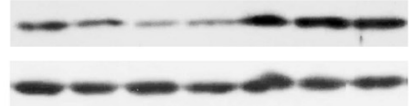

Contralateral
$\mathbf{F}$

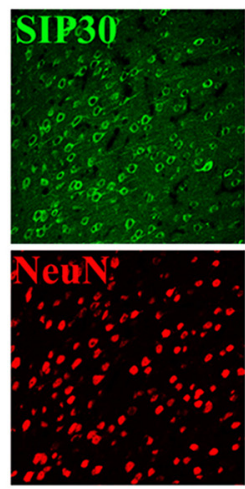

SIP30/NeuN

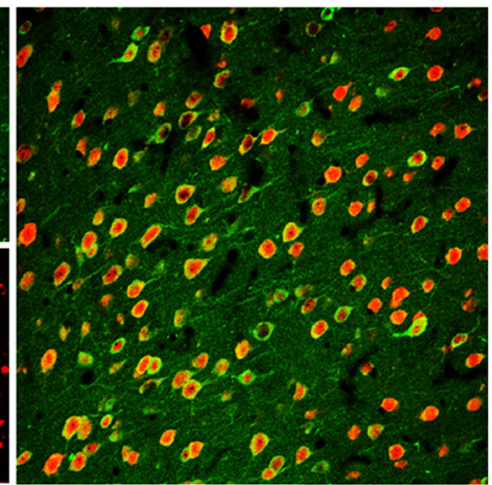

SIP30/GFAP
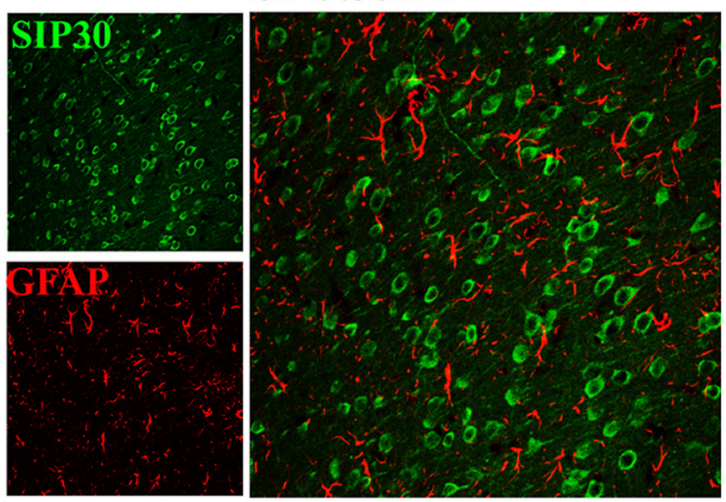

SIP30/OX-42
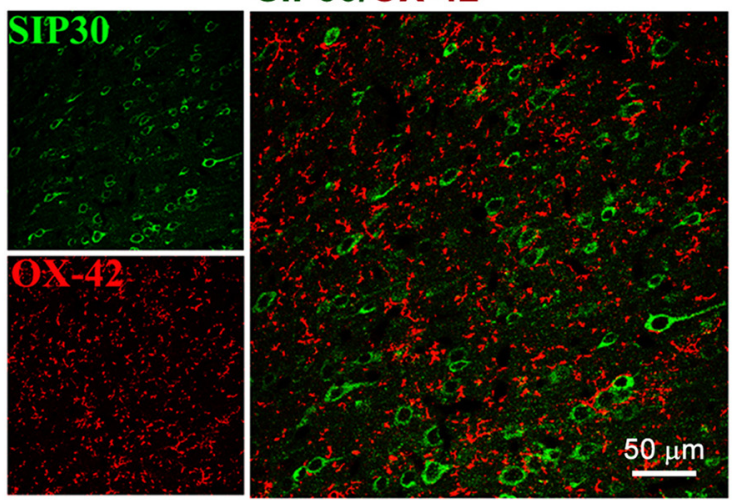

Figure 1. SIP30 expression in the $\mathrm{ACCC} . \boldsymbol{A}, \boldsymbol{B}$, Robust thermal hyperalgesia $(\boldsymbol{A})$ and mechanical allodynia $(\boldsymbol{B})$ were observed in the ipsilateral hindpaw after $C(\mathrm{Cl}$. $\boldsymbol{C}, \boldsymbol{D}$, Western blot analyses reveal an increase in SIP30 levels in the bilateral rACC after CCI. GAPDH served as a loading control. SIP30 levels are normalized against GAPDH levels. ${ }^{* *} p<0.01$ vs sham. $\boldsymbol{E}$, Left, Schematic drawing indicating different laminae of the rACC. Right, A low-magnification section showing SIP30 immunoreactivity in laminae II-VI of the rACC. $F$, Double staining showing that SIP30 colocalizes with NeuN (a neuronal marker), but does not colocalize with GFAP (an astrocytic marker) or OX-42 (a microglial marker) in naive rats.

SIP30 knockdown eliminated neuropathic pain-evoked aversion by reducing glutamate release in the $\mathrm{ACC}$

The pain-evoked PEAP was used to evaluate emotional/affective responses to neuropathic pain. As shown in Figure $2 A$, CCI rats spent significantly more time on the light side of the chamber, suggesting that these animals were willing to avoid a preferred area (dark side) to escape stimulation of the affected paw. Control animals preferred to remain in the dark and tended to avoid the light side of the chamber (two-way ANOVA; treatment, $F_{(1,13)}=$ 93.932, $p<0.01$ ).

To determine the contribution of SIP30 to pain-related aversion, lentivirus expressing SIP30-shRNA was intra-rACC injected into the rACC to knock down SIP30. Western blot analyses showed that SIP30 expression was successfully suppressed 7 and
$14 \mathrm{~d}$ after SIP30-shRNA treatment (Fig. 2B). Fluorescence-tagged SIP30 shRNA exhibited a similar profile. SIP30 immunoreactivity in the rACC was robustly reduced by SIP30-shRNA (Fig. 2C). The CCI rats that received a bilateral intra-rACC injection of SIP30-shRNA $\left(1.5 \times 10^{9} \mathrm{TU} / \mathrm{ml}\right)$ spent significantly less time on the light side of the chamber compared with rats that received shNC injection (two-way ANOVA; treatment, $F_{(2,28)}=63.163$, $p<0.01)$. In the sham-CCI rats, SIP30-shRNA had no effect on PEAP (Fig. 2D). Pretreatment with SIP30-shRNA on day 7 before CCI prevented CCI-induced SIP30 upregulation in the rACC (one-way ANOVA, $F_{(3,20)}=38.904, p<0.01$; Fig. $2 E$ ).

Our previous study found that knockdown of SIP30 in PC12 cells reduces the neurotransmitter pool available for synaptic vesicle exocytosis, suggesting that SIP30 may participate in the 
modulation of neurotransmitter release (Zhang et al., 2009). To determine whether SIP30 is involved in presynaptic glutamate release, we recorded mEPSCs in rACC slices. No significant differences in frequencies of mEPSCs were detected between the naive rats and rats transfected with shNC. However, in the SIP30shRNA-treated rats, frequencies of mEPSCs were significantly reduced compared with naive and shNC-treated rats (oneway ANOVA, $\left.F_{(2,53)}=5.953, p<0.01\right)$, although the amplitudes of the mEPSCs were not altered (Fig. 3A-E). We also used EPSC PPRs as another indicator of presynaptic glutamate release. At stimulus intervals ranging from 35 to $250 \mathrm{~ms}$, the PPRs of the SIP30-shRNA-treated rats were increased compared with those of the shNC-treated rats (Fig. 3F). Because PPRs are inversely related to release probability (Zucker and Regehr, 2002), our results indicate that knockdown of SIP30 decreased presynaptic glutamate release. Furthermore, we also measured the concentrations of glutamate in the extracellular dialysates of the rACC. At a flow rate of $2 \mu \mathrm{l} / \mathrm{min}$, the base dialysate concentration of glutamate was $2.54 \pm 0.17 \mu \mathrm{M}(n=33)$ in the rACC. In the shNC-treated rats, CCI induced an increase in extracellular glutamate at days 3 and 7. Following SIP30 knockdown, CCI-induced upregulation of extracellular glutamate was completely prevented (Fig. 3G). Additionally, extracellular glutamate concentrations were significantly decreased by intrarACC injection of SIP30-shRNA in both the sham and CCI groups (two-way ANOVA; Groups: $F_{(3,28)}=13.202, p<$ 0.01 ; Group $\times$ Time interaction: $F_{(9,84)}=$ $2.085, p=0.037)$. Intra-rACC injection of homocystic acid (HCA; $100 \mathrm{~mm}, 0.6 \mu \mathrm{l}$ per side), an ionotropic glutamate receptor agonist, rescued the impairments in pain-related escape/avoidance in the SIP30shRNA-treated CCI rats (two-way ANOVA, Groups: $F_{(2,20)}=155.837, p<0.01$; Group $\times$ Time interaction: $F_{(10,200)}=$ $2.837, p<0.01)$. In the shNC-treated controls, HCA did not affect CCI-induced pain-related avoidance (Fig. $3 H$ ).

\section{SIP30 knockdown did not affect CCI-} induced behavioral hypersensitivity or general learning and memory

To determine whether downregulation of SIP30 in the rACC influenced basal nociceptive threshold and CCI-induced painlike behavioral hypersensitivity, we measured PWTs in response to Von Frey hairs, and PWLs in response to radiant heat stimulation. Intra-rACC injection of shNC or shRNA had no effect on basal PWTs or PWLs. Unilateral CCI induced profound thermal hyperalgesia and mechanical allodynia on day 7 in the ipsilateral
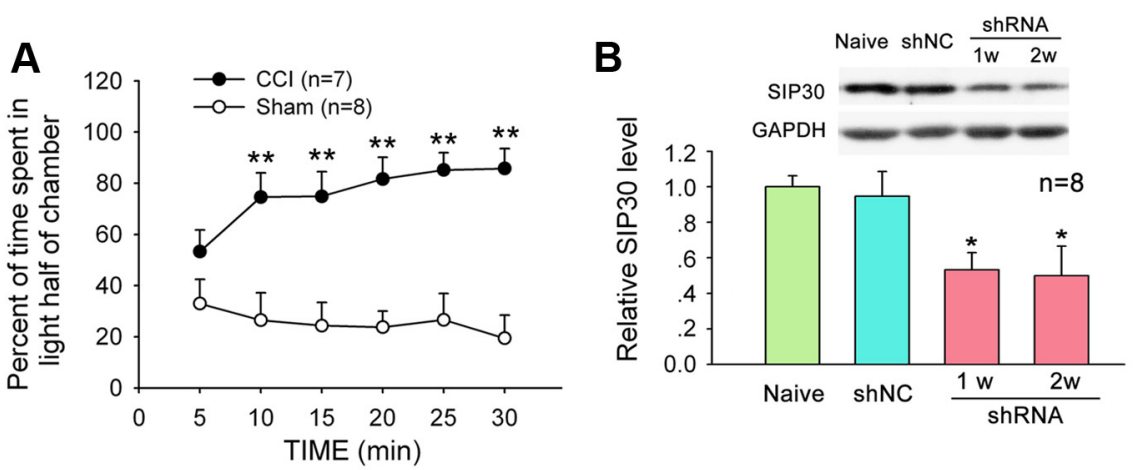

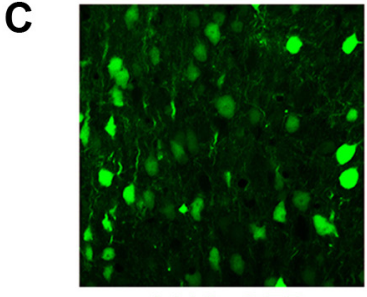

shNC-GFP

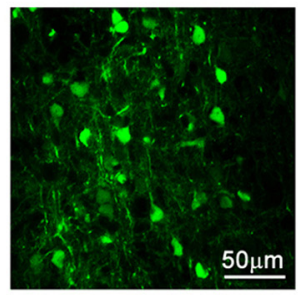

shRNA-GFP

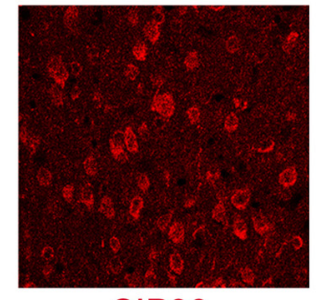

SIP30

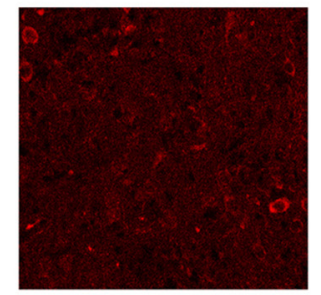

SIP30

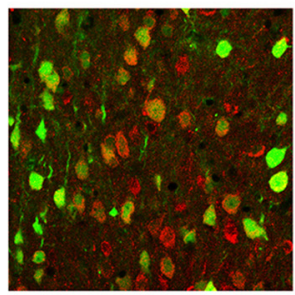

shNC-GFP/SIP30

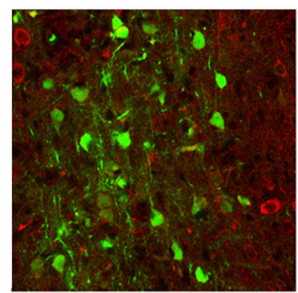

shRNA-GFP/SIP30

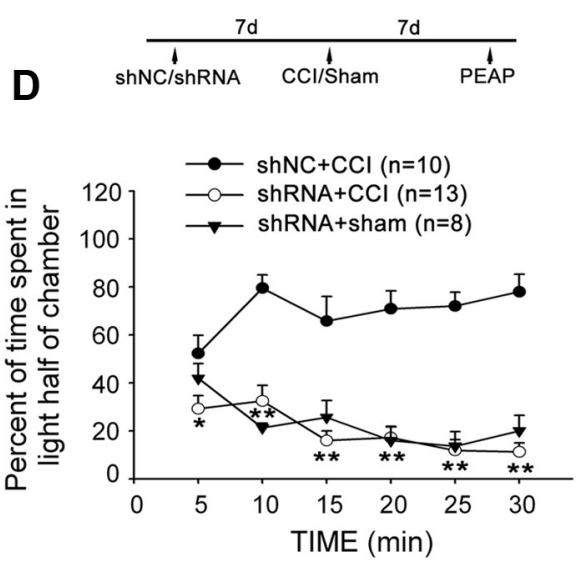

\section{E}
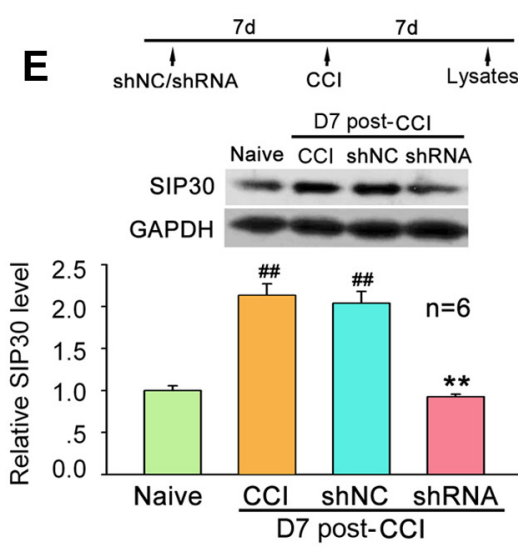

Figure 2. SIP30 contributes to neuropathic pain-evoked aversion. $A, C(I$ rats spent significantly more time on the light side of the chamber in the PEAP test, indicating that these animals were willing to avoid a preferred area (dark area) to escape stimulation of the affected paw. Control animals preferred to remain in the dark and tended to avoid the light side of the chamber. ${ }^{* *} p<0.01$ vs sham control. $\boldsymbol{B}$, Western blots showing successful suppression of SIP30 in the rACC at 7 and $14 \mathrm{~d}$ after intra-rACC SIP30-shRNA lentivirus. SIP30 levels are normalized against GAPDH levels. ${ }^{*} p<0.05$ vs shNC. C, The efficiency of SIP30 knockdown was confirmed by immunofluorescence. SIP30 immunoreactivity in the rACC was robustly reduced by SIP30-shRNA but not by shNC. $D$, Downregulation of SIP30 by shRNA in the rACC prevented PEAP induction. Top, The protocol for behavioral testing. ${ }^{*} p<0.05$, ${ }^{* *} p<0.01$ vs shNC. $\boldsymbol{E}$, Intra-rACC SIP30-shRNA blocked CCI-induced SIP30 upregulation. SIP30 levels are normalized against GAPDH levels. Top, A schematic of the experimental design. ${ }^{* *} p<0.01$ vs shNC; ${ }^{\# \#} p<0.01$ vs naive.

hindpaw. CCI-induced hyperalgesia and allodynia were not affected by SIP30 knockdown in the rACC (Fig. 4A, B).

To determine whether SIP30 in rACC is specific to painrelated aversive learning, we also assessed the effects of SIP30 knockdown on general neural processing related to learning and memory. We first examined CPA induced by an aversive agent ( $\mathrm{LiCl}, 150 \mathrm{mg} / \mathrm{kg}$, i.p.). As shown in Figure 4C, when systemic 
A

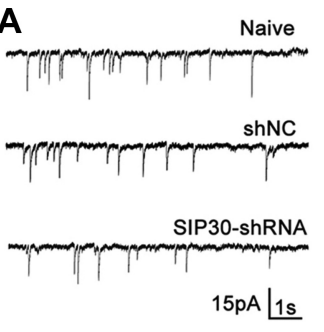

D

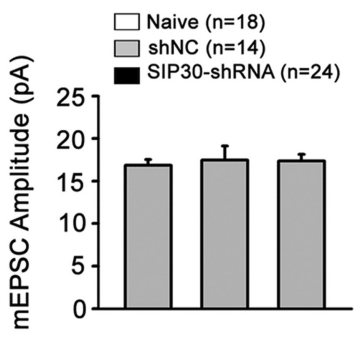

B

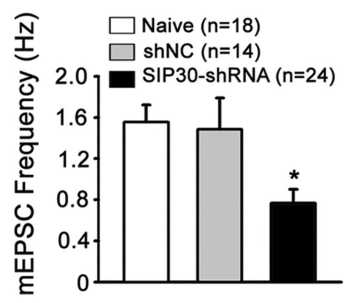

E

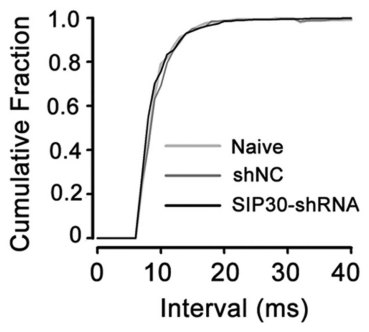

C

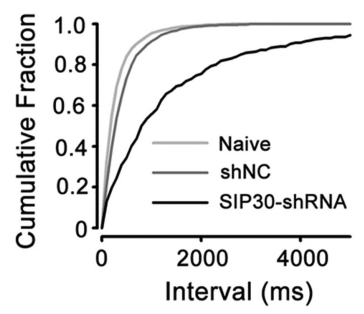

$\mathbf{F}$
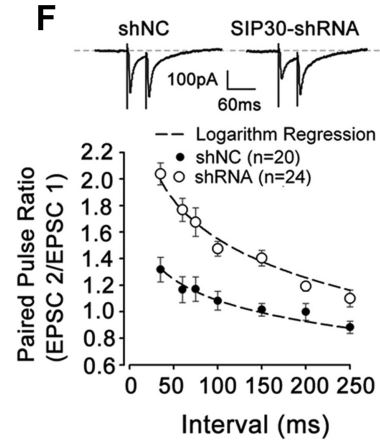

We further examined the role of SIP30 in the rACC during spatial learning and memory using the Morris water maze. As shown in Figure 4E, both groups of rats that received shNC or SIP30-shRNA were able to learn to find the submerged platform in the Morris water maze; the escape latencies became shorter with increased numbers of training trials in both groups (two-way ANOVA, $F_{(1,14)}=0.011, p=$ $0.918)$. Moreover, when memory retention was tested $24 \mathrm{~h}$ after training, the SIP30-shRNA-treated rats also displayed escape latencies that were similar to those of the shNC-treated rats (Fig. 4E,F). Additionally, no deficits in performance on the visible platform test were found in either group (Fig. 4G).

\section{ERK and PKA regulated SIP30 expression in the rACC}

In a previous study, we demonstrated that ERK activation promotes the recruitment of the transcription factor CREB to the sip30 gene promoter in PC12 cells (Peng et al., 2009). To determine whether ERK activation is required for CCI-induced SIP30 upregulation in the rACC, PD98059 (1 nmol), an MEK inhibitor, was microinjected into the bilateral rACC every other day for a total of four injections, the first of which occurred $30 \mathrm{~min}$ before CCI. CCI-induced SIP30 increases in the rACC were significantly prevented by the MEK inhibitor PD98059 (Fig. 5A). Additionally, intra-rACC administration of RpcAMP (1 nmol), a PKA inhibitor, produced an effect on SIP30 expression that was similar to that of PD98059 (oneway ANOVA, $\left.F_{(2,18)}=9.183, p=0.002\right)$. Accordingly, PEAP testing showed that both PD98059- and Rp-cAMP-treated CCI rats spent significantly less time on the light side of the chamber than did vehicle-treated rats (10\% DMSO) on day 7 (two-way ANOVA; Groups: $F_{(2,17)}=$ 129.892, $p<0.01$; Fig. 5B).

Western blot analyses indicated that pERK1/2, pPKA, and PCREB were obviously increased on days 7 and 14 after CCI (Fig. 5C-E), which was associated with CCI-induced SIP30 upregulation. Double-

injection of $\mathrm{LiCl}$ was paired with a particular compartment in the place-conditioning apparatus, CPA was elicited. Moreover, this LiCl-induced CPA was not affected by SIP30 knockdown (one-way ANOVA, $F_{(2,19)}=0.702, p=0.508$ ). Next, we examined the effect of SIP30 knockdown on footshock-induced fear conditioning. Like $\mathrm{LiCl}$ injection, low-intensity electric footshocks $(0.5 \mathrm{~mA}, 2 \mathrm{~s})$, which failed to evoke nociceptive responses in spinal dorsal horn neurons (Gao et al., 2004), produced CPA [electric footshock-induced CPA (S-CPA)]. Interestingly, S-CPA was also SIP30-independent because the downregulation of SIP30 in the bilateral rACC had no effect on S-CPA (Fig. 4D). immunofluorescence staining indicated that nearly all of the pCREB-positive cells were SIP30-positive cells and that most of the pCREB-positive cells expressed pPKA substrate. Furthermore, pERK was exclusively expressed in pCREB- and SIP30positive cells in the rACC (Fig. $5 F$ ).

\section{Discussion}

SIP30, which was first identified in the cochlear hair cells of the guinea pig organ of Corti (Lee et al., 2002), is expressed in many organs but is most abundant in the brain (Peng et al., 2009; Zhang et al., 2009). However, the molecular and cellular functions of 

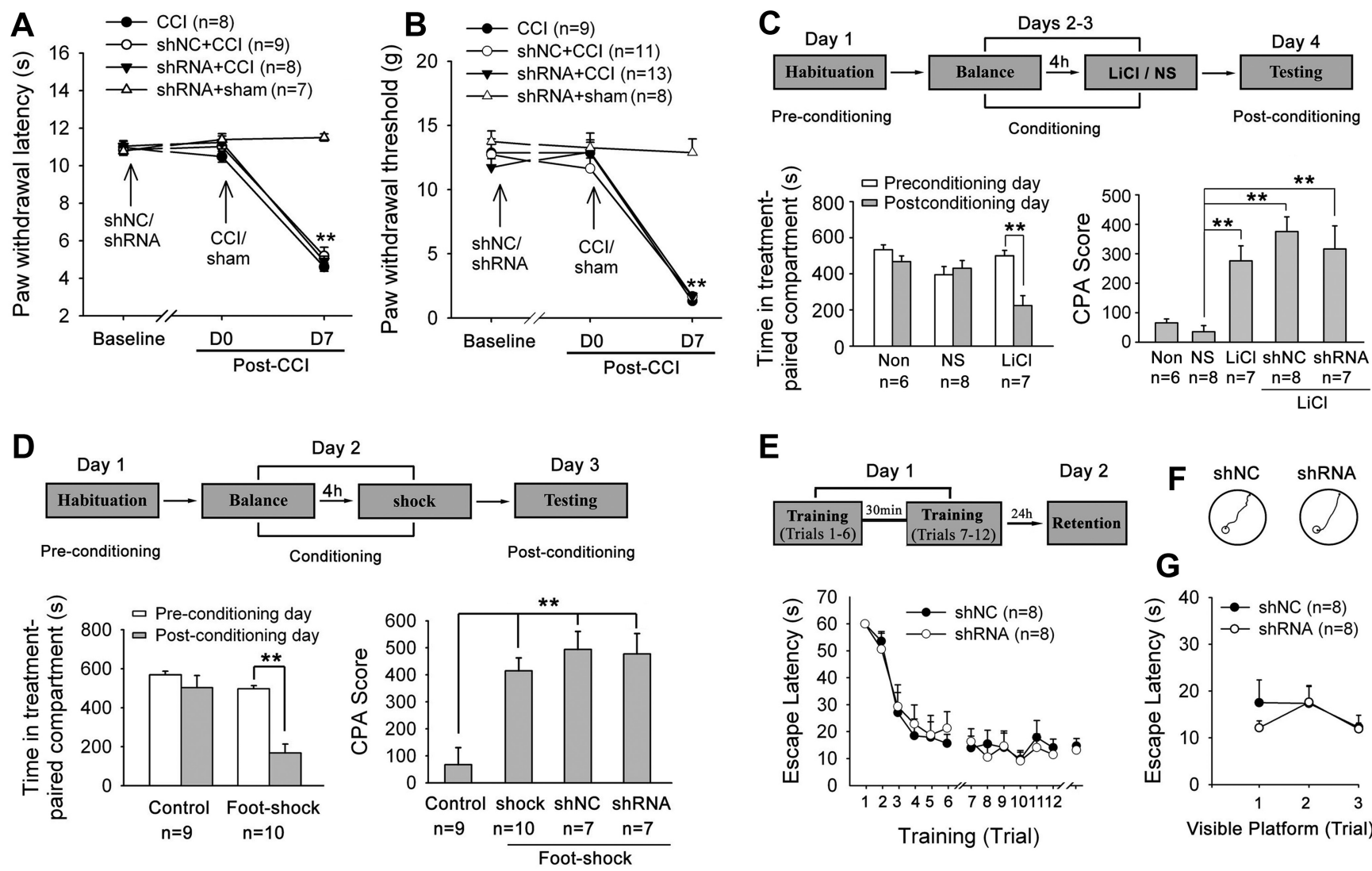

Figure 4. Downregulation of SIP30 in the rACC does not alter CCl-induced hypersensitivity or general learning and memory. $\boldsymbol{A}, \boldsymbol{B}$, Intra-rACC SIP30-shRNA did not suppress CCI-induced thermal hyperalgesia $(\boldsymbol{A})$ or mechanical allodynia $(\boldsymbol{B}) .{ }^{* *} p<0.01$ vs sham. $\boldsymbol{C}, \boldsymbol{D}$, Downregulation of SIP30 in the rACC did not block LiCl-induced CPA (C) or electric footshock-induced CPA (D), as indicated by the time the animals spent in the treatment-paired compartment on the preconditioning and postconditioning days (left) and the CPA scores (right). Top, The protocol for behavioral testing. ${ }^{* *} p<0.01$. $\boldsymbol{E}-\boldsymbol{G}$, Downregulation of SIP30 did not impair spatial learning and memory in the Morris water maze. $\boldsymbol{E}$, Escape latencies (i.e., the times required to find the submerged platform). The cutoff time was $60 \mathrm{~s}$ and shNC or SIP30-shRNA was given 2 weeks before training in the Morris water maze, which consisted of two training sessions of six trials each with a 30 min resting period between the two sessions. Memory retention was tested $24 \mathrm{~h}$ after training. The retention values are calculated as the mean of three-trial retention tests. Top, Illustrates the behavioral testing protocol. $\boldsymbol{F}$, Representative swimming traces of a shNC- and a shRNA-treated rat during the $24 \mathrm{~h}$ retention test. $\boldsymbol{G}$, Escape latencies. The platform was raised above the turbid liquid surface and was visible.

SIP30 remain elusive. Our previous studies indicated that SIP30 is required in the spinal cord for the development and the maintenance of the neuropathic pain induced by CCI (Zhang et al., 2009). The present study further suggests that SIP30 in the rACC is critical for peripheral nerve injury-induced affective pain.

\section{SIP30 in the rACC contributes to affective pain}

Increasing evidence indicates that the rACC has important roles in pain-related negative emotion and pain-related aversive learning in humans and animals (Rainville et al., 1997; Johansen et al., 2001; Pereira et al., 2013). Previous studies from our laboratory and other groups have demonstrated that formalin-induced CPA, which is believed to reflect the emotional component of pain, and PEAP, another behavioral test that quantifies the level of aversion evoked by painful stimuli, depends on the activity in the rACC (Lei et al., 2004; LaGraize and Fuchs, 2007; Li et al., 2009; Uhelski and Fuchs, 2009; Xiao et al., 2013). Our current study showed that SIP30 was upregulated following the development of CCI-induced neuropathic pain and that blockade of SIP30 upregulation prevented pain-related aversive behavior, suggesting a causal relationship between SIP30 upregulation and the development of negative emotion in neuropathic pain conditions.

Given that the PEAP is based on associative learning between painful stimulus-induced aversion and environmental context, the inhibition of pain-related aversive behavior may be caused by a disruption of neural processing related to learning and memory. Our data showed that neither was the ability of the animal to acquire CPA induced by non-nociceptive fear conditioning and an aversive agent $(\mathrm{LiCl})$ nor was spatial learning/memory in the Morris water testing impaired by SIP30 knockdown. It is noteworthy that although S-CPA has been used routinely as a fearconditioning task, the noxious or innocuous components elicited by the footshock have been seldom discussed. Our previous test demonstrated that the $0.5-10 \mathrm{~mA}$ electric stimulation applied to the paw plantar surface failed to evoke nociceptive C-responses in spinal dorsal horn neurons (Gao et al., 2004). Even in CCI rats, the threshold of electrical shock-induced nociceptive C-responses in spinal wide dynamic range neurons ranges from 5 to $12 \mathrm{~mA}$ (data not shown). It is conceivable that the electric shock $(0.5 \mathrm{~mA})$ used in the present study was not noxious stimulation. Thus, $0.5 \mathrm{~mA}$ footshock-induced avoidance behavior might implicate a complicated constellation of responses. This can explain why knockdown of SIP30 in the ACC blocked pain-related PEAP, but did not affect footshock-induced CPA. Consistently, previous findings from our group and other investigators indicate that neither footshock-induced (Gao et al., 2004) nor U69,593-induced (an aversive agent; Johansen et al., 2001) CPA was blocked by ACC lesions. Furthermore, cocaine-induced conditioned place preference was not blocked by ACC lesions (Qu et al., 2011). It is 
A

\begin{tabular}{ccccc}
$\downarrow_{30 \mathrm{~min}}$ & $\downarrow$ & $\downarrow$ & $\downarrow$ & $\mathrm{D} 7$ \\
\hline $\mathrm{D} 0$ & $\mathrm{D} 2$ & $\mathrm{D} 4$ & $\mathrm{D} 6$ & $\begin{array}{c}\uparrow \\
\text { LCI }\end{array}$ \\
& & &
\end{tabular}

B

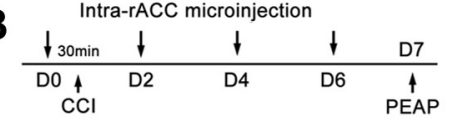

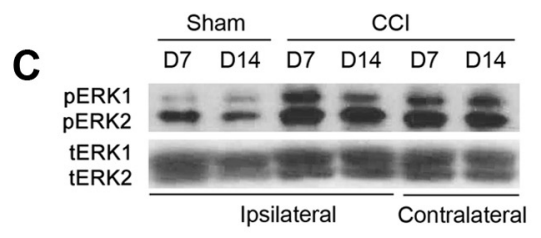
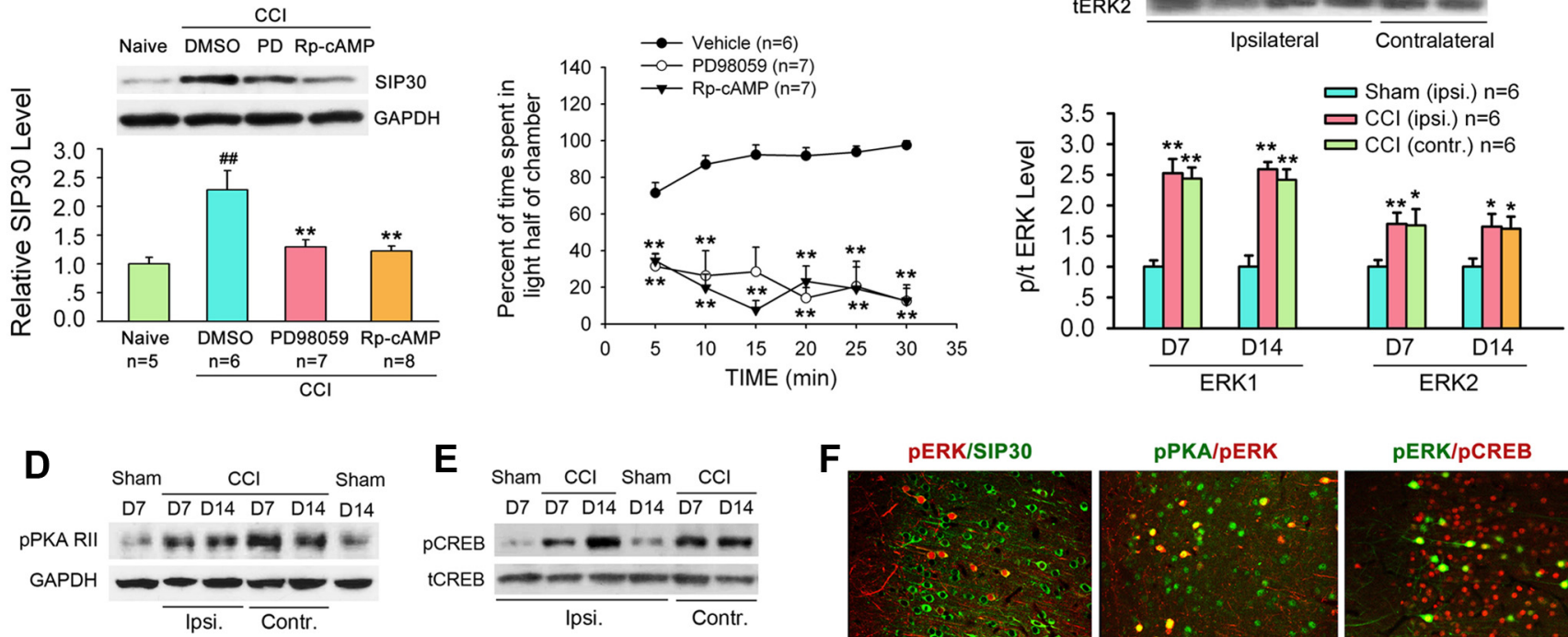

$\mathbf{E}$
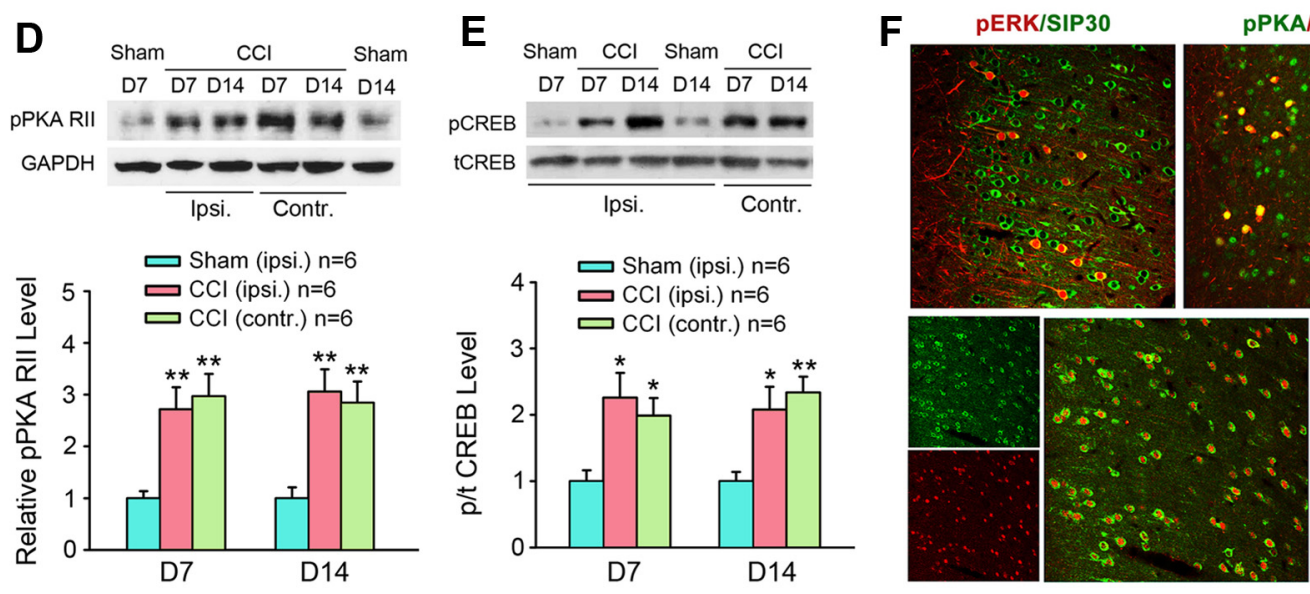

pCREB/SIP30

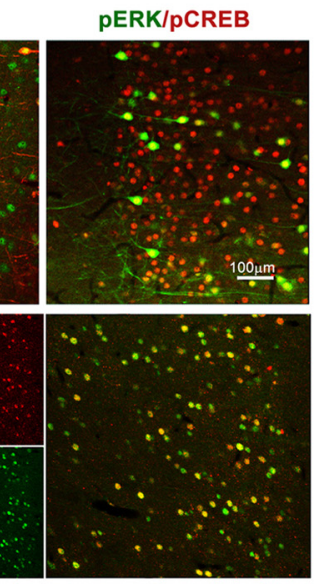

teax

pPKA/pCREB

Figure 5. ERK and PKA signaling controls SIP30 expression in the rACC. A, CCI-induced SIP30 upregulation was blocked by intra-rACC injection of the MEK inhibitor PD98059 or the PKA inhibitor Rp-cAMP. SIP30 levels are normalized against GAPDH levels. Top, A schematic of the experimental design. ${ }^{* *} p<0.01$ vs DMSO (vehicle); ${ }^{\# \#} p<0.01$ vs naive. B, Blockade of ERK or PKA activation in the rACC prevented PEAP induction. Top, The protocol for behavioral testing. ${ }^{* *} p<0.01$ vs vehicle. $(-\boldsymbol{E}$, Western blot analyses reveals increased pERK (C), pPKA (D), and pCREB $(\boldsymbol{E})$ levels in the rACC after CCI. pERK1 and pERK2 levels are normalized against total ERK1 and ERK2 levels; pPKA RII levels are normalized against GAPDH levels; $p$ CREB levels are normalized against total CREB levels. ${ }^{*} p<0.05,{ }^{* *} p<0.01$ vs sham. $\boldsymbol{F}$, Double immunofluorescence revealed the colocalization of pERK with $p$ PKA, pCREB, and SIP30, and the colocalization of pCREB with pPKA and SIP30.

reasonable to assume that SIP30 upregulation in the rACC is particularly important for pain-related negative emotion and pain-related aversive learning and general learning processes.

SIP30 upregulation in the spinal cord after CCI has been shown to contribute to the development of neuropathic pain (Zhang et al., 2009). To determine whether SIP30 upregulation in the rACC is also important for neuropathic pain, we examined the effect of downregulation of SIP30 on CCI-induced hyperalgesia and allodynia. In support of earlier studies from our and other groups showing that either bilateral lesions of the rACC or blockade of ERK activation in the rACC does not inhibit nerve injury-induced neuropathic pain (Cao et al., 2009; Qu et al., 2011), the present study showed that CCI-induced hyperalgesia and allodynia were not affected by knockdown of SIP30 in the rACC.

\section{SIP30 in the rACC regulates glutamate release}

SIP30 was first characterized as a protein that interacts with SNAP25. A major function of SNAP25 is the regulation of synaptic vesicle exocytosis and neurotransmitter release (Nagy et al., 2004). The other SNAP25 interacting proteins have been re- ported to be involved in synaptic vesicle exocytosis (Ilardi et al., 1999; Tian et al., 2005). Therefore, SIP30 is likely involved in modulating neurotransmitter levels. In a previous study, we found that inhibition of SIP30 by siRNA in PC12 cells cotransfected with a human growth hormone plasmid significantly reduced basal and total vesicular hormone secretion (Zhang et al., 2009), suggesting that SIP30 may participate in modulating steady-state neurotransmitter levels in the packed synaptic vesicles that are available for exocytosis. The present results further showed reduced mEPSC frequencies and increased PPRs in rACC slices from SIP30-shRNA-treated rats, suggesting that downregulation of SIP30 may decrease presynaptic glutamate release. The extracellular concentration of glutamate was consistently and significantly decreased following knockdown of SIP30 in the rACC. Notably, intra-rACC injection of SIP30-shRNA not only prevented CCI-induced upregulation of extracellular glutamate but also reduced basal extracellular glutamate concentrations in sham rats, indicating that SIP30 may be involved in both basal and stimulated glutamate release. Together, SIP30 may contribute to the development of affective pain by regulating glutamate release in the rACC during neuropathic pain. Consis- 
tently, Xu et al. (2008) reported that the presynaptic release probability of glutamate is enhanced in rACC neurons after nerve injury.

\section{Activation of ERK and PKA mediates SIP30 upregulation in the rACC}

Our previous study demonstrated that activation of ERK and PKA in the rACC is required for the development of pain-related negative emotion (Cao et al., 2009, 2012). Activation of ERK and PKA has been implicated in CREB-mediated gene transcription (Impey et al., 1998). CREB is known to play an important role in transducing pain signals and mediating long-term plasticity (Hoeger-Bement and Sluka, 2003; Ran et al., 2012). Phosphorylated CREB can bind to the promoters of many genes to regulate their transcription. In the NGF-stimulated cell model, we found that CREB-mediated ERK-regulated sip30 expression and that NGF promoted the recruitment of CREB to the sip30 promoter (Peng et al., 2009). There are several soluble NSF attachment protein receptor family members, such as SYN3 and SNAP25, that are downstream targets of CREB (Euskirchen et al., 2004). It is possible the sip30 functions as an ERK/PKA-regulated CREB target in CCI-induced pain-related aversive behaviors. Our present data showed that pERK and pCREB were largely colocalized with SIP30 and that pERK or pPKA was present with PCREB in the rACC neurons after CCI. CCI-induced upregulation of SIP30 correlated with ERK, PKA, and CREB activation in the rACC. When ERK or PKA activation was inhibited, the CCI-induced SIP30 upregulation was significantly suppressed. Moreover, inhibition of ERK or PKA had inhibitory effects on neuropathic pain-induced aversive behavior that were similar to those induced by knockdown of SIP30. This finding provides a potential therapeutic strategy for the development of drugs that can interfere with SIP30 expression to attenuate pain-related negative emotion without affecting ERK and PKA activity.

In conclusion, we identified a SNAP25-associated protein, SIP30, as an important player in neuropathic pain-evoked aversion in the CCI model. Inhibition of SIP30 expression in the rACC specifically suppressed CCI-induced aversion but did not affect CCI-induced hypersensitivity or general learning and memory. CCI-induced SIP30 upregulation depended on PKA or/and ERK activation. Thus, SIP30, as a potential downstream target of PKA and ERK, contributes to the induction of the painrelated negative emotion induced by peripheral nerve injury.

\section{References}

Auvray M, Myin E, Spence C (2010) The sensory-discriminative and affective-motivational aspects of pain. Neurosci Biobehav Rev 34:214223. CrossRef Medline

Bennett MK, Calakos N, Scheller RH (1992) Syntaxin: a synaptic protein implicated in docking of synaptic vesicles at presynaptic active zones. Science 257:255-259. CrossRef Medline

Cao H, Gao YJ, Ren WH, Li TT, Duan KZ, Cui YH, Cao XH, Zhao ZQ, Ji RR, Zhang YQ (2009) Activation of extracellular signal-regulated kinase in the anterior cingulate cortex contributes to the induction and expression of affective pain. J Neurosci 29:3307-3321. CrossRef Medline

Cao H, Ren WH, Zhu MY, Zhao ZQ, Zhang YQ (2012) Activation of glycine site and GluN2B subunit of NMDA receptors is necessary for ERK/CREB signaling cascade in rostral anterior cingulate cortex in rats: implications for affective pain. Neurosci Bull 28:77-87. CrossRef Medline

Euskirchen G, Royce TE, Bertone P, Martone R, Rinn JL, Nelson FK, Sayward F, Luscombe NM, Miller P, Gerstein M, Weissman S, Snyder M (2004) CREB binds to multiple loci on human chromosome 22. Mol Cell Biol 24:3804-3814. CrossRef Medline

Frisch C, Hasenöhrl RU, Mattern CM, Häcker R, Huston JP (1995) Blockade of lithium chloride-induced conditioned place aversion as a test for antiemetic agents: comparison of metoclopramide with combined ex- tracts of Zingiber officinale and Ginkgo biloba. Pharmacol Biochem Behav 52:321-327. CrossRef Medline

Fuchs PN, McNabb CT (2012) The place escape/avoidance paradigm: a novel method to assess nociceptive processing. J Integr Neurosci 11:6172. CrossRef Medline

Gao YJ, Ren WH, Zhang YQ, Zhao ZQ (2004) Contributions of the anterior cingulate cortex and amygdala to pain- and fear-conditioned place avoidance in rats. Pain 110:343-353. CrossRef Medline

Hoeger-Bement MK, Sluka KA (2003) Phosphorylation of CREB and mechanical hyperalgesia is reversed by blockade of the cAMP pathway in a time-dependent manner after repeated intramuscular acid injections. J Neurosci 23:5437-5445. Medline

Ilardi JM, Mochida S, Sheng ZH (1999) Snapin: a SNARE-associated protein implicated in synaptic transmission. Nat Neurosci 2:119-124. CrossRef Medline

Impey S, Obrietan K, Wong ST, Poser S, Yano S, Wayman G, Deloulme JC, Chan G, Storm DR (1998) Cross talk between ERK and PKA is required for $\mathrm{Ca} 2+$ stimulation of CREB-dependent transcription and ERK nuclear translocation. Neuron 21:869-883. CrossRef Medline

Johansen JP, Fields HL (2004) Glutamatergic activation of anterior cingulate cortex produces an aversive teaching signal. Nat Neurosci 7:398-403. CrossRef Medline

Johansen JP, Fields HL, Manning BH (2001) The affective component of pain in rodents: direct evidence for a contribution of the anterior cingulate cortex. Proc Natl Acad Sci U S A 98:8077-8082. CrossRef Medline

LaGraize SC, Fuchs PN (2007) GABA(A) but not GABA(B) receptors in the rostral anterior cingulate cortex selectively modulate pain-induced escape/avoidance behavior. Exp Neurol 204:182-194. CrossRef Medline

Lee HK, Safieddine S, Petralia RS, Wenthold RJ (2002) Identification of a novel SNAP25 interacting protein (SIP30). J Neurochem 81:1338-1347. CrossRef Medline

Lei LG, Sun S, Gao YJ, Zhao ZQ, Zhang YQ (2004) NMDA receptors in the anterior cingulate cortex mediate pain-related aversion. Exp Neurol 189: 413-421. CrossRef Medline

Li TT, Ren WH, Xiao X, Nan J, Cheng LZ, Zhang XH, Zhao ZQ, Zhang YQ (2009) NMDA NR2A and NR2B receptors in the rostral anterior cingulate cortex contribute to pain-related aversion in male rats. Pain 146:183193. CrossRef Medline

Marie-Claire C, Courtin C, Robert A, Gidrol X, Roques BP, Noble F (2007) Sensitization to the conditioned rewarding effects of morphine modulates gene expression in rat hippocampus. Neuropharmacology 52:430-435. CrossRef Medline

Mochida S (2000) Protein-protein interactions in neurotransmitter release. Neurosci Res 36:175-182. CrossRef Medline

Mutso AA, Radzicki D, Baliki MN, Huang L, Banisadr G, Centeno MV, Radulovic J, Martina M, Miller RJ, Apkarian AV (2012) Abnormalities in hippocampal functioning with persistent pain. J Neurosci 32:5747-5756. CrossRef Medline

Nagy G, Reim K, Matti U, Brose N, Binz T, Rettig J, Neher E, Sørensen JB (2004) Regulation of releasable vesicle pool sizes by protein kinase A-dependent phosphorylation of SNAP-25. Neuron 41:417-429. CrossRef Medline

Paxinos G, Watson C (1998) The rat brain in sterotaxic coordinates. New York: Academic.

Peng G, Han M, Du Y, Lin A, Yu L, Zhang Y, Jing N (2009) SIP30 is regulated by ERK in peripheral nerve injury-induced neuropathic pain. J Biol Chem 284:30138-30147. CrossRef Medline

Pereira EA, Boccard SG, Moir L, Fitzgerald JJ, Green AL, Aziz TZ (2013) Deep brain stimulation of the anterior cingulate cortex: targeting the affective component of chronic pain. Paper presented at 2013 Congress of Neurological Surgeons, San Francisco, October.

Price DD (2000) Psychological and neural mechanisms of the affective dimension of pain. Science 288:1769-1772. CrossRef Medline

Qu C, King T, Okun A, Lai J, Fields HL, Porreca F (2011) Lesion of the rostral anterior cingulate cortex eliminates the aversiveness of spontaneous neuropathic painfollowing partial or complete axotomy. Pain 152: 1641-1648. CrossRef Medline

Rainville P, Duncan GH, Price DD, Carrier B, Bushnell MC (1997) Pain affect encoded in human anterior cingulate but not somatosensory cortex. Science 277:968-971. CrossRef Medline

Ran I, Laplante I, Lacaille JC (2012) CREB-dependent transcriptional con- 
trol and quantal changes in persistent long-term potentiation in hippocampal interneurons. J Neurosci 32:6335-6350. CrossRef Medline

Ren WH, Guo JD, Cao H, Wang H, Wang PF, Sha H, Ji RR, Zhao ZQ, Zhang YQ (2006) Is endogenous D-serine in the rostral anterior cingulate cortex necessary for pain-related negative affect? J Neurochem 96:16361647. CrossRef Medline

Rinaman L, Saboury M, Litvina E (2009) Ondansetron blocks LiCl-induced conditioned place avoidance but not conditioned taste/flavor avoidance in rats. Physiol Behav 98:381-385. CrossRef Medline

Söllner T, Whiteheart SW, Brunner M, Erdjument-Bromage H, Geromanos S, Tempst P, Rothman JE (1993) SNAP receptors implicated in vesicle targeting and fusion. Nature 362:318-324. CrossRef Medline

Tian JH, Wu ZX, Unzicker M, Lu L, Cai Q, Li C, Schirra C, Matti U, Stevens D, Deng C, Rettig J, Sheng ZH (2005) The role of Snapin in neurosecretion: snapin knock-out mice exhibit impaired calcium-dependent exocytosis of large dense-core vesicles in chromaffin cells. J Neurosci 25:10546-10555. CrossRef Medline

Uhelski ML, Fuchs PN (2009) Naltrexone fails to increase pain affect in response to inflammatory pain in a novel escape/avoidance paradigm. Physiol Behav 98:263-267. CrossRef Medline
Xiao X, Yang Y, Zhang Y, Zhang XM, Zhao ZQ, Zhang YQ (2013) Estrogen in the anterior cingulate cortex contributes to pain-related aversion. Cereb Cortex 23:2190-2203. CrossRef Medline

Xu H, Wu LJ, Wang H, Zhang X, Vadakkan KI, Kim SS, Steenland HW, Zhuo M (2008) Presynaptic and postsynaptic amplifications of neuropathic pain in the anterior cingulate cortex. J Neurosci 28:7445-7453. CrossRef Medline

Yu J, Lou GD, Yue JX, Tang YY, Hou WW, Shou WT, Ohtsu H, Zhang SH, Chen Z (2013) Effects of histamine on spontaneous neuropathic pain induced by peripheral axotomy. Neurosci Bull 29:261-269. CrossRef Medline

Zhang YQ, Guo N, Peng G, Wang X, Han M, Raincrow J, Chiu CH, Coolen LM, Wenthold RJ, Zhao ZQ, Jing N, Yu L (2009) Role of SIP30 in the development and maintenance of peripheral nerve injury-induced neuropathic pain. Pain 146:130-140. CrossRef Medline

Zimmermann M (1983) Ethical guidelines for investigations of experimental pain in conscious animals. Pain 16:109-110. CrossRef Medline

Zucker RS, Regehr WG (2002) Short-term synaptic plasticity. Annu Rev Physiol 64:355-405. CrossRef Medline 